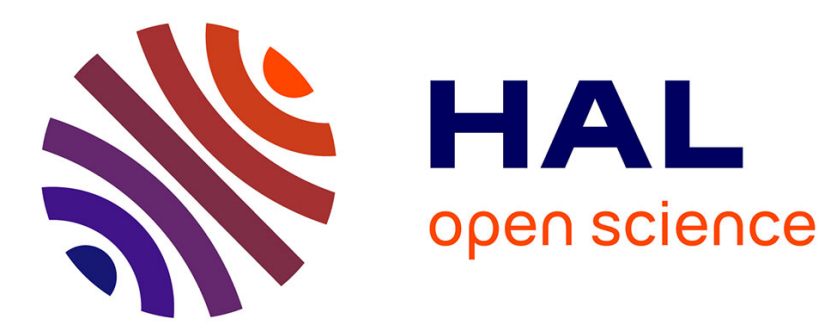

\title{
Alin: improving interactive ontology matching by interactively revising mapping suggestions
}

Jomar da Silva, Kate Revoredo, Fernanda Araujo Baião, Jérôme Euzenat

\section{To cite this version:}

Jomar da Silva, Kate Revoredo, Fernanda Araujo Baião, Jérôme Euzenat. Alin: improving interactive ontology matching by interactively revising mapping suggestions. Knowledge Engineering Review, 2020, 35, pp.e1. 10.1017/S0269888919000249 . hal-02984949

\section{HAL Id: hal-02984949 \\ https://hal.science/hal-02984949}

Submitted on 1 Nov 2020

HAL is a multi-disciplinary open access archive for the deposit and dissemination of scientific research documents, whether they are published or not. The documents may come from teaching and research institutions in France or abroad, or from public or private research centers.
L'archive ouverte pluridisciplinaire HAL, est destinée au dépôt et à la diffusion de documents scientifiques de niveau recherche, publiés ou non, émanant des établissements d'enseignement et de recherche français ou étrangers, des laboratoires publics ou privés. 


\title{
ALin: Improving Interactive Ontology Matching by Interactively Revising Mapping Suggestions
}

\author{
Jomar da Silva $^{1}$, Kate Revoredo ${ }^{2}$, Fernanda Baião ${ }^{3}$, Jérôme Euzenat ${ }^{4}$ \\ ${ }^{1}$ Graduate Program in Informatics, Federal University of Rio de Janeiro (UFRJ), Brazil \\ E-mail: jomar.silva@uniriotec.br \\ ${ }^{2}$ Graduate Program in Informatics, Federal University of Rio de Janeiro (UFRJ), Brazil \\ E-mail: katerevoredo@ppgi.ufrj.br \\ ${ }^{3}$ Department of Informatics, Pontifical Catholic University of Rio de Janeiro (PUC-Rio), Brazil \\ E-mail: fbaiao@inf.puc-rio.br \\ ${ }^{4}$ Univ. Grenoble Alpes, INRIA, CNRS, Grenoble INP, LIG, F-38000 Grenoble, France \\ E-mail: Jerome.Euzenat@inria.fr
}

\begin{abstract}
Ontology matching aims at discovering mappings between the entities of two ontologies. It plays an important role in the integration of heterogeneous data sources that are described by ontologies. Interactive ontology matching involves domain experts in the matching process. In some approaches, the expert provides feedback about mappings between ontology entities, i.e., these approaches select mappings to present to the expert who replies which of them should be accepted or rejected, so taking advantage of the knowledge of domain experts towards finding an alignment. In this paper, we present ALIN, an interactive ontology matching approach which uses expert feedback not only to approve or reject selected mappings, but also to dynamically improve the set of selected mappings, i.e., to interactively include and to exclude mappings from it. This additional use for expert answers aims at increasing in the benefit brought by each expert answer. For this purpose, Alin uses four techniques. Two techniques were used in previous versions of Alin to dynamically select concept and attribute mappings. Two new techniques are introduced in this paper: one to dynamically select relationship mappings and another one to dynamically reject inconsistent selected mappings using anti-patterns. We compared ALIN with state-of-theart tools, showing that it generates alignment of comparable quality.
\end{abstract}

\section{Introduction}

An ontology is a formal and explicit artifact that represents a shared conceptualization on a particular domain, structurally consisting of a collection of interconnected entities (concepts, attributes of concepts and relationships between concepts). One advantage of the use of ontologies is to improve communication, not only among humans but also among application systems using these ontologies. This, in turn, fosters interoperability. However, due to the recent and steeply increasing advancements of the semantic technologies and the web, there are scenarios in which several ontologies exist for the same domain, each using different entities to refer to the same realworld object. These scenarios raise communication issues among people or application systems that use these different ontologies.

Ontology matching has successfully addressed these problems by discovering correspondences (mappings) between entities of different ontologies (Euzenat and Shvaiko, 2013). One of the possible approaches to the ontology matching process is the interactive one that involves domain experts in the matching process. This involvement can be used to improve the results over fully automatic approaches (Paulheim et al., 2013). 


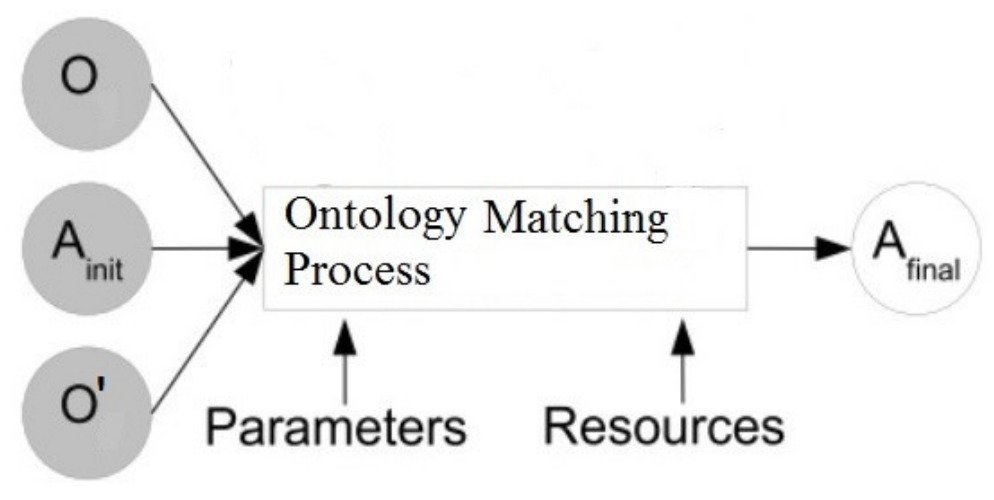

Figure 1 Ontology Matching Process - based on (Euzenat and Shvaiko, 2013)

In some interactive approaches, the domain expert provides feedback about mappings between ontology entities. These approaches select mappings to present to the expert who assesses which of them should be accepted or rejected. They take advantage of the domain expert knowledge to generate an alignment, and have, as their two main steps, the selection of the mappings to receive expert feedback and the propagation of this feedback. These approaches vary in techniques for these two steps.

Current approaches select mappings before beginning the propagation step, i.e., they do not select new mappings during the propagation step. The problem with these approaches is that they do not take advantage of the expert's knowledge to select new mappings.

We focused on this problem, the first time, with $A L I N_{\text {Syn }}$ (Da Silva et al., 2017), which interactively selected concept mappings. In a second time, we developed a new technique, which interactively selected attribute mappings, which gave rise to the other approach, ALIN $N_{\text {Attr }}$ (Da Silva, Revoredo, Baião and Euzenat, 2018). Alin uses the two techniques implemented in $A L I N_{S y n}$ and $A L I N_{A t t r}$.

The contributions of this research are the definition of a new structural technique to interactively select new relationship mappings and the interactive use of anti-patterns, so using the expert feedback to improve the set of selected mappings.

The Ontology Alignment Evaluation Initiative (OAEI) is a coordinated international initiative whose one of the goals is to assess the strengths and weaknesses of ontology matching systems. ALIN has participated in OAEI 2018 and was evaluated in the interactive matching track, with the Conference and Anatomy data sets. When compared with the other tools, Alin got the highest precision in both data sets (Algergawy et al., 2018). In the Conference track, Alin achieved the highest recall among all tools. On the Anatomy track, Alin has not reached a prominent recall, for reasons this paper will explain.

The rest of this paper is organized as follows. Section 2 reviews Interactive Ontology Matching. In Section 3, we present the related works. Section 4 fully details Alin algorithms. Section 5 describes our evaluation methodology and discusses experimental results. Finally, Section 6 concludes the paper.

\section{Interactive Ontology Matching}

The ontology matching process starts with two ontologies, $O$ and $O^{\prime}$, and returns an alignment $A_{\text {final }}$ between the input ontologies. An alignment is a set of mappings between entities $e$ and $e^{\prime}$ from $O$ and $O^{\prime}$ respectively, defining pairs of entities. The ontology matching process can receive as input an initial alignment $A_{\text {init }}$. Besides, it can get input parameters and can access external resources, such as a dictionary (Figure 1).

A mapping asserts a semantic relationship between its entities, such as disjunction, subsumption, or equivalence. In this paper, we only consider the equivalence relationship. The ontology 


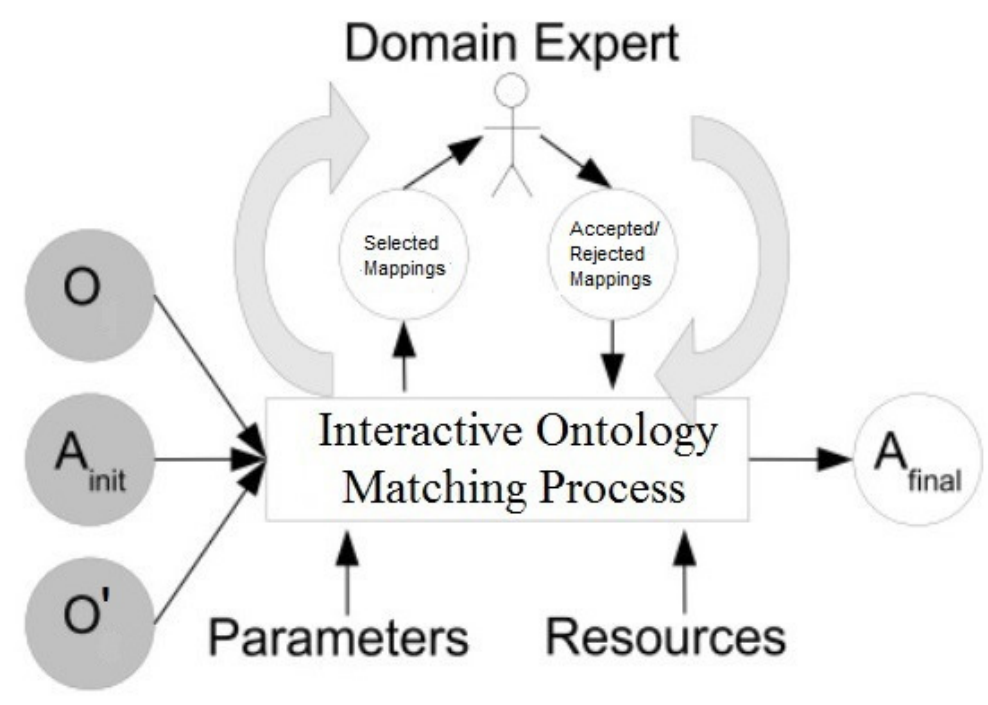

Figure 2 Interactive Ontology Matching Process - based on (Paulheim et al., 2013 )

matching system is responsible to find, among all the possible mappings, the ones to be included in the alignment.

Given that two ontologies representing the same domain may present different types of heterogeneity (terminological, structural or semantic, among others), we need a combination of distinct techniques to correctly discover mappings between their entities. We can see an ontology matching tool as a collection of several matching components, each implementing a specific technique dealing with a specific heterogeneity type (Ngo et al., 2013).

Interactive ontology matching processes involve domain experts. The type of expert involvement used in this paper is through feedback on selected mappings. This type takes benefit from the domain expert knowledge by submitting selected mappings to the expert who assesses which of them should be accepted or rejected (Figure 2).

One common type of approach for this type of expert involvement is to divide the process into two main steps: the selection of mappings and the propagation of expert feedback. The current tools that follow this type of approach only select mappings in the selection step. They do not select any new mappings in the propagation step, thus not taking advantage of the expert feedback to select mappings.

This paper presents an approach where various techniques are used to use the expert feedback to improve the set of selected mappings, including a new technique for interactively select relationship mappings, and also including the interactive use of mapping anti-patterns.

This paper shows that the new technique causes an increase in F-measure, and, if the approach uses expert feedback as the input of the technique, then the approach requires fewer interactions with the expert to do so. It also shows that the interactive use of mapping anti-patterns decrease the number of interactions with the expert.

\section{Related Work}

There are at least three areas in which users may be involved in a matching solution (Euzenat and Shvaiko, 2013):

- by providing initial alignments to the system (before matching),

- by configuring (which includes strategy and parameter selection) and tuning the system, and

- by providing feedback to matchers (during or after the automatic matching process) in order for them to adapt their results. 
The type of user, a domain expert, involvement used in this paper is through feedback to matchers. They benefit from the domain expert knowledge by submitting selected mappings to the expert who assesses which of the mappings should be accepted or rejected. One common type of approach for this type of expert involvement is to divide the process into two main steps: the selection of mappings and the propagation of expert feedback.

In the propagation step, different approaches can use expert feedback in different ways. Some of these approaches automatically classify selected mappings using a threshold. The threshold is a value that indicates whether a mapping should be automatically accepted (or rejected) when its similarity values are greater (or smaller) than the threshold. Expert feedback is used to calculate this threshold (Paulheim and Hertling, 2013; Hertling, 2012; Duan et al., 2010; Shi et al., 2009 Chunhua et al., 2015). Some approaches automatically classify some selected mappings using a classifier. These approaches use expert feedback to create the training data set for learning the classifiers (Lopes et al., 2015, To H. et al., 2009). Others approaches use expert feedback to modify the weight of similarity metrics (Duan et al. 2010; Shi et al. 2009; Balasubramani et al. 2015) or to change the value of similarity metrics (Cruz et al. 2012, 2014).

Alin and its previous versions use the expert feedback to improve the set of selected mappings. Others approaches also use expert feedback to improve the set of selected mappings (Lambrix and Kaliyaperumal, 2016, Jiménez-Ruiz et al., 2012, Faria et al., 2013.

$A L I N_{S y n}$ (Da Silva et al., 2017), an earlier version of ALIN, in the mapping selection phase, selects concept mappings through semantic and lexical similarity metrics. Afterward, it automatically accepts the selected concept mappings with the same names. Then, it suspends some of these mappings using a semantic technique. In the propagation phase, $A L I N_{S y n}$ uses expert feedback to unsuspend some of these suspended mappings, returning them to the set of selected mappings, thus improving it. ALIN uses all the techniques used in $A L I N_{S y n}$.

$A L I N_{A t t r}$ (Da Silva, Revoredo, Baião and Euzenat, 2018), another earlier version of Alin, has the same mapping selection phase as $A L I N_{S y n}$, but without suspending selected concept mappings. In the programming phase, $A L I N_{A t t r}$ uses expert feedback to select attribute mappings. ALIN uses the attribute mapping selection technique used in $A L I N_{A t t r}$.

SAMBO (Lambrix and Kaliyaperumal, 2016) takes advantage of the expert feedback to improve the set of selected mappings, removing the selected mappings that conflict with the accepted mappings, according to a reasoner.

Logmap (Jiménez-Ruiz and Grau, 2011, Jiménez-Ruiz et al., 2012), in the selection step, select mappings based on similarity metrics seeking to achieve a high recall. Still, in this step, the approach also uses automatic, non-interactive, techniques for improving the precision of the set of selected mappings. In the propagation step, the approach takes advantage of the expert feedback by identifying mappings that are inconsistent with the accepted mappings, according to a reasoner, and removes them from the set of selected mappings.

AML (Faria et al., 2013) initially selects mappings based on lexical, semantic and structural similarities. AML places, in automatic mode, mappings that are above a given threshold in the alignment, which then goes through a repair process.

In the interactive mode, AML uses two thresholds. Those selected mappings that are below the lower threshold, are automatically rejected. Selected mappings above the higher threshold are automatically accepted unless they generate an inconsistency. In such a case, they are submitted to the expert. Concerning the selected mappings between the two thresholds, those which generate inconsistency are automatically rejected; the others are submitted to the expert.

AML uses the expert feedback to remove mappings from the set of selected mappings. If a selected mappings is accepted, all selected mappings that have an entity in common with it are rejected, as well as all those that are inconsistent with it.

In the current approaches, the impact of expert feedback on the set of selected mappings is the removal of mappings from it, to increase the precision and decrease the number of interactions. 
ALIN and its previous versions also focus on using the expert feedback to select mappings to improve the set of selected mappings.

Alin uses, besides the techniques of the earlier versions, another that selects relationships mappings associated with accepted concept mappings, and mapping anti-patterns.

In the approach of (Chunhua et al. 2015), there are formulas that associate relationships mappings to the concept mappings of the concepts associated with these relationships. The formulas serve to increase the weight of these concept mappings, which increases the likelihood of the approach automatically include them into the final alignment. ALIN selects relationships mappings, to receive the expert feedback, associated with accepted concepts mappings.

ALIN uses mapping anti-patterns to remove mappings from the set of selected mappings. ASMOV (Jean-Mary et al., 2009) uses concepts similar to mapping anti-patterns, which it calls semantic verification inferences. ASMOV chooses from the mappings found in one of these semantic verification inferences to take out of the alignment. ASMOV is an automatic matching tool, so unlike Alin, it does not use expert feedback to identify which mappings to exclude from alignment.

Section 4 further explains the ALIN approach.

Several areas use user knowledge to improve their performance. In information search and retrieval area, for example, feedback data based on direct interaction (e.g., clicks, scrolling) as well as on user profiles/preferences have been proven valuable for personalizing the search process, e.g., from how queries are understood to how relevance is assessed (Belkin et al., 2010). However, in such cases, the feedback is provided by users and is used for answering the query of the same user (in a single session for relevance feedback, or across session for personalization). Ontology matching could take advantage of user feedback to improve alignments. However, interactive ontology matching is usually disconnected from the use of the resulting alignments, which may be used in different contexts. It relies on expert feedback, as opposed to user feedback, in order to find the correct alignment, not one that suits the need of a particular user. In addition, relevance feedback in information retrieval is rather used to give more or less weight to characteristics of the instances. Here it is used to revise the mappings based on more logical constraint propagation, e.g., anti-patterns.

\section{The Alin Approach}

In this section, we describe our approach for interactively matching two ontologies. The next subsection presents the overall ALIN procedure. Section 4.2 presents the terminology used to explain our approach. Section 4.3 describe the Alin algorithms.

\subsection{General principles}

ALIN handles three sets of mappings:

Accepted definitely to be retained in the alignment

Selected to be decided

Suspended selected but filtered out

The procedure of Alin is:

1. Select mappings: select the first mappings;

2. Filter mappings: suspend some selected mappings, using semantic criteria for that;

3. Ask expert: accepts or rejects selected mappings

4. Propagate: select new mappings, reject some selected mappings or unsuspend some suspended mappings (depending on newly accepted mappings)

5. Go back to 3 as long as there are undecided selected mappings 
All versions of ALIN follow this general scheme. They mainly differ on the way they implement the Propagation step.

There were two previous versions of ALIN: $A L I N_{S y n}$, and $A L I N_{A t t r}$. The problem addressed by these versions was to improve the set of selected mappings using expert feedback to select better mappings to present to the expert. These versions differ from the type of mappings that is presented to the expert: $A L I N_{\text {Syn }}$, uses concept mappings only, and $A L I N_{A t t r}$, uses attribute mappings in addition.

One contribution of this paper, concerning the previous ALIN versions, is a new structural technique to select relationship mappings, implemented by the SelectRelationshipMappings algorithm (Algorithm 9) used in the Propagate phase. The goal of the new technique is to increase the F-measure, and if the expert feedback is used to assist it, do it more efficiently (more increase per provided feedback).

In order to show the benefits of exploiting expert feedback to choose questions, we have developed two new versions of ALIN: ALIN NoRel and ALIN $N_{\text {Aut }}$.

Another contribution is the use of mapping anti-patterns to reject the selected mappings that are inconsistent with the mappings accepted by the expert. Alin uses various algorithms to avoid and undo these anti-patterns (Algorithms 10, 11, 12 and 13).

To identify the benefits of the interactive use of anti-patterns, we have developed another version of ALIN: $A L I N_{N o A P}$.

\subsection{Terminology}

In an ontology $O$, its entities $e$ can be concepts (c), which are concepts in a domain, organized in a subconcept-superconcept hierarchy, or properties, which describe attributes $(a)$ of concepts and relationships $(r)$ between concepts. Table 1 provides predicates that can be used to model an ontology.

Table 1 Predicates for ontology description (based on (Chunhua et al., 2015))

\begin{tabular}{cc}
\hline Predicate & Description \\
\hline \hline $\operatorname{concept}(c, O)$ & $c$ is a concept of ontology $O$ \\
\hline $\operatorname{attr}(a, O)$ & $a$ is an attribute of ontology $O$ \\
\hline $\operatorname{relat}(r, O)$ & $r$ is a relationship of ontology $O$ \\
\hline $\operatorname{entity}(e, O)$ & $e$ is an entity of ontology $O$ \\
\hline $\operatorname{sub}\left(c_{1}, c_{2}\right)$ & $c_{1}$ is subconcept of $c_{2}$ \\
\hline $\operatorname{sub}\left(c_{1}, c_{2}, \mathrm{n}\right)$ & $c_{1}$ is subconcept of $c_{2}$ with a maximum of $\mathrm{n}$ depth levels \\
\hline $\operatorname{dis}\left(c_{1}, c_{2}\right)$ & $c_{1}$ is disjoint of $c_{2}$ \\
\hline $\operatorname{rconcept}\left(\mathrm{r}, c_{1}, c_{2}\right)$ & $r$ is a relationship between $c_{1}$ and $c_{2}$ \\
\hline aconcept $(a, c)$ & $a$ is an attribute of concept $c$ \\
\hline
\end{tabular}

Given two ontologies $O$ and $O^{\prime}$, an ontology matching approach will search for mappings between the entities of these two ontologies. In this paper, all entities from $O$ have no prime $\left(^{\prime}\right)$; all entities from $O^{\prime}$ have a prime $\left(^{\prime}\right)$. We use the notation $\left\langle e, e^{\prime}\right\rangle$ to represent a mapping between two entities, $\left\langle c, c^{\prime}\right\rangle$ between two concepts, and $\left\langle r, r^{\prime}\right\rangle$ and $\left\langle a, a^{\prime}\right\rangle$ between two relationships and two attributes.

\subsection{Algorithms}

The Alin algorithms (Algorithms 1 to 14 use the variables described below:

- Selected - Set of selected mappings;

- Suspended - Set of suspended mappings; 
- Accepted - Set of accepted mappings;

- SetofSimMet - Set of similarity metrics;

- SetofSemSim - Set of semantic similarity metrics, it is a subset of the set of similarity metrics;

- SimMet - one similarity metric;

Alin uses the functions defined in Table 2 in its algorithms.

Table 2 Functions used in the Alin algorithms

\begin{tabular}{cl}
\hline Function & \multicolumn{1}{c}{ Description } \\
\hline \hline ename $\left(e_{1}, e_{2}\right)$ & returns true if the name of entity $e_{1}$ is equal to the \\
& name of entity $e_{2}$, else return false. \\
\hline highsum $(A)$ & returns the mapping from the set of mappings $A$ with \\
& the highest sum of similarity metrics. \\
\hline concepts $(O)$ & returns the set of all concepts of the ontology O. \\
\hline attributes $(c)$ & returns the set of all attributes of the concept c. \\
\hline multent $\left(\left\langle e_{1}, e_{1}^{\prime}\right\rangle,\left\langle e_{2}, e_{2}^{\prime}\right\rangle\right)$ & returns true if $\left\langle e_{1}, e_{1}^{\prime}\right\rangle$ and $\left\langle e_{2}, e_{2}^{\prime}\right\rangle$ are in a multiple- \\
& entity anti-pattern, else it returns false. See Defini- \\
& tion 4.2. \\
\hline antipat $\left(\left\langle e_{1}, e_{1}^{\prime}\right\rangle,\left\langle e_{2}, e_{2}^{\prime}\right\rangle\right)$ & returns true if $\left\langle e_{1}, e_{1}^{\prime}\right\rangle$ and $\left\langle e_{2}, e_{2}^{\prime}\right\rangle$ are in any of \\
& the three anti-patterns, else it returns false. See \\
& Definitions 4.2 to 4.4 \\
\hline simvalue $\left(\left\langle e, e^{\prime}\right\rangle\right.$, SimMet $\left.^{\prime}\right)$ & returns the value of the metric SimMet between the \\
& entities e and e'. \\
\hline isconcmap $\left(\left\langle e, e^{\prime}\right\rangle\right)$ & returns true if e and e' are concepts, else it returns \\
& false. \\
\hline isattrmap $\left(\left\langle e, e^{\prime}\right\rangle\right)$ & returns true if e and e' are attributes, else it returns \\
& false. \\
\hline isrelatmap $\left(\left\langle e, e^{\prime}\right\rangle\right)$ & returns true if e and e' are relationships, else it \\
& returns false. \\
\hline isaccepted $\left(\left\langle e, e^{\prime}\right\rangle\right)$ & returns true if the expert accepts the mapping $\left\langle e, e^{\prime}\right\rangle$, \\
& else it returns false. \\
\hline trigger $\left(\left\langle c_{1}, c_{1}^{\prime}\right\rangle,\left\langle c_{2}, c_{2}^{\prime}\right\rangle,\left\langle r, r^{\prime}\right\rangle\right)$ & returns true if there is a trigger, between $\left\langle c_{1}, c_{1}^{\prime}\right\rangle$ and \\
& $\left\langle c_{2}, c_{2}^{\prime}\right\rangle$, for the selection of $\left\langle r, r^{\prime}\right\rangle$, else it returns false. \\
& See Definition 4.1 \\
\hline
\end{tabular}

Alin (Algorithm 1) has two main steps: the selection step and the propagation step. The selection step is responsible for defining the initial selected, accepted and suspended mappings. The propagation step is where the expert provides feedback to the selected mappings and this feedback is propagated.

The following subsections detail these two steps.

\subsubsection{Selection Step}

The ALIN selection step (from line 1 to line 3 of Algorithm 1) describes the activities for defining the initial set of selected mappings, the initial set of suspended mappings and the initial alignment (accepted mappings). The algorithm has as input the two ontologies to be matched and, as parameters, a set of similarity metrics chosen by the user. The chosen similarity metrics can be semantic or lexical.

The algorithm starts selecting concept mappings (line 1 of Algorithm 1) using the SelectConceptMappings algorithm (Algorithm 2). Inside this algorithm, the SelectMappingsPerMetric algorithm (Algorithm 3) selects mappings for each similarity metric. The SelectMappingsPerMetric algorithm treats the matching problem as a stable marriage problem with size list limited 


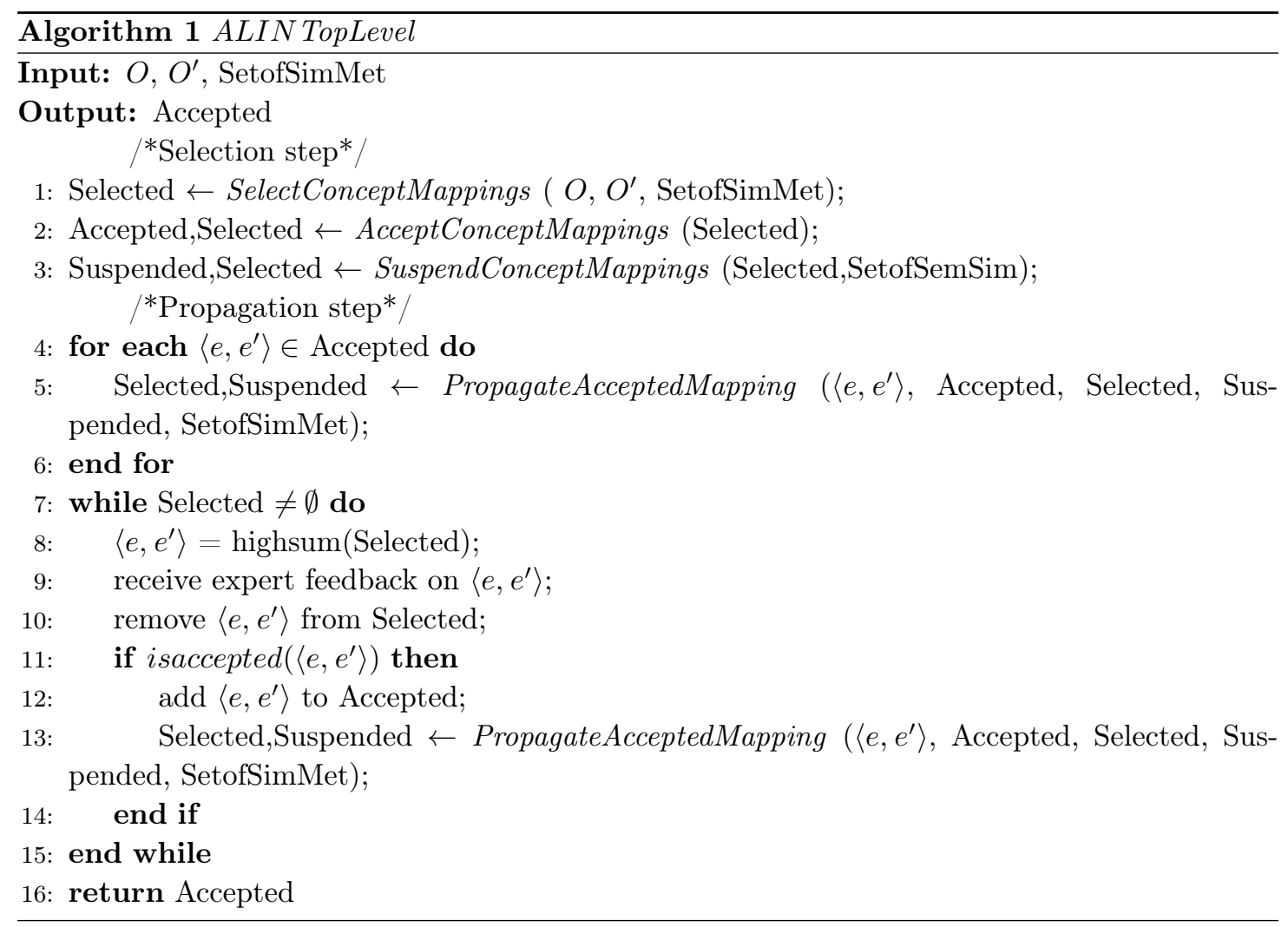

to 1 (Gale and Shapley, 1962, Irving et al., 2009), i.e., the algorithm only selects one mapping if similarity value between the two entities of the mapping is the highest considering all the mappings with at least one of these entities.

The SelectMappingsPerMetric algorithm is executed once for each chosen metric, where it generates a set of mappings for each metric (lines 4 and 5 of Algorithm 2). The union of these sets defines the initial set of selected mappings (from line 6 to line 10 of Algorithm 2 .

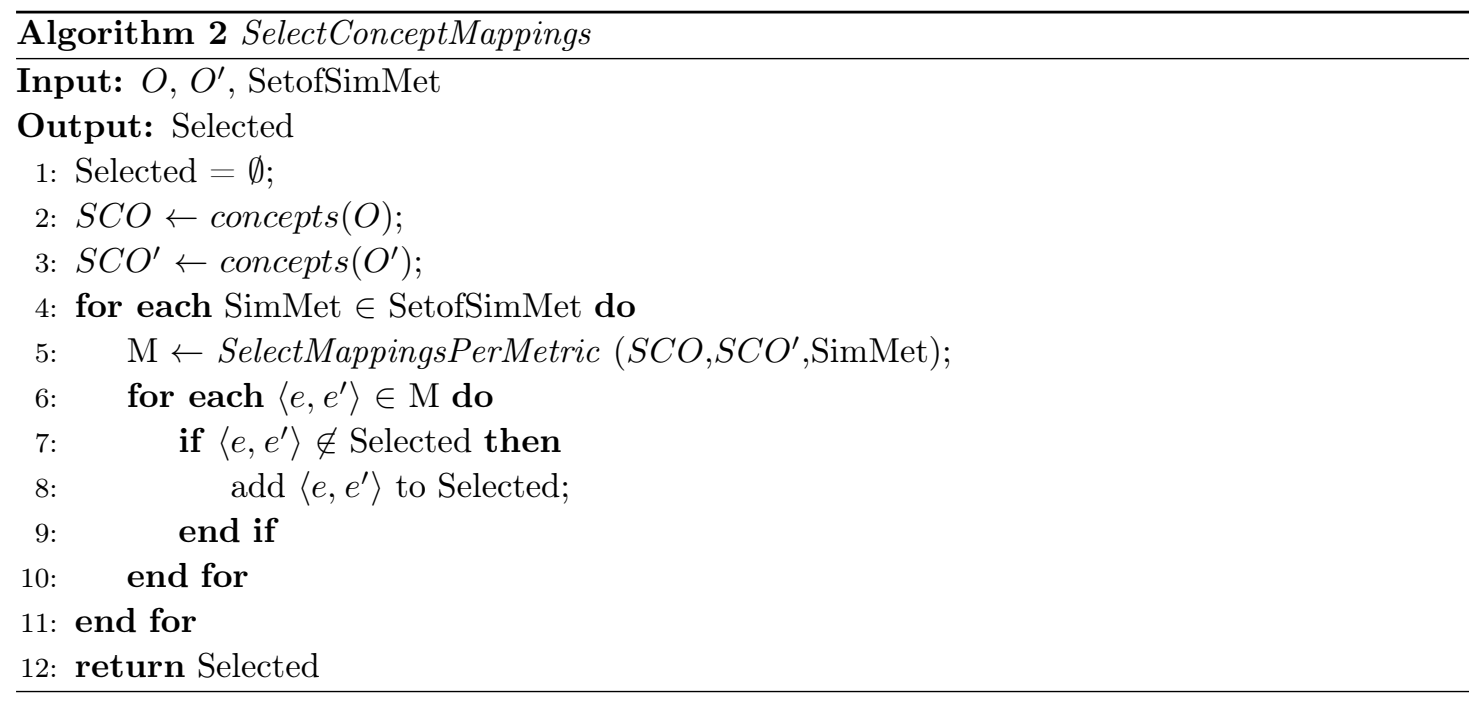

After defining an initial set of selected mappings, ALIN evaluates each of them to verify if some of them can already be automatically accepted (line 2 of Algorithm 1) using the AcceptConceptMappings algorithm (Algorithm 4). At first, ALIN automatically accepts concept mappings whose entity names are the same and moves them from the set of selected mappings 


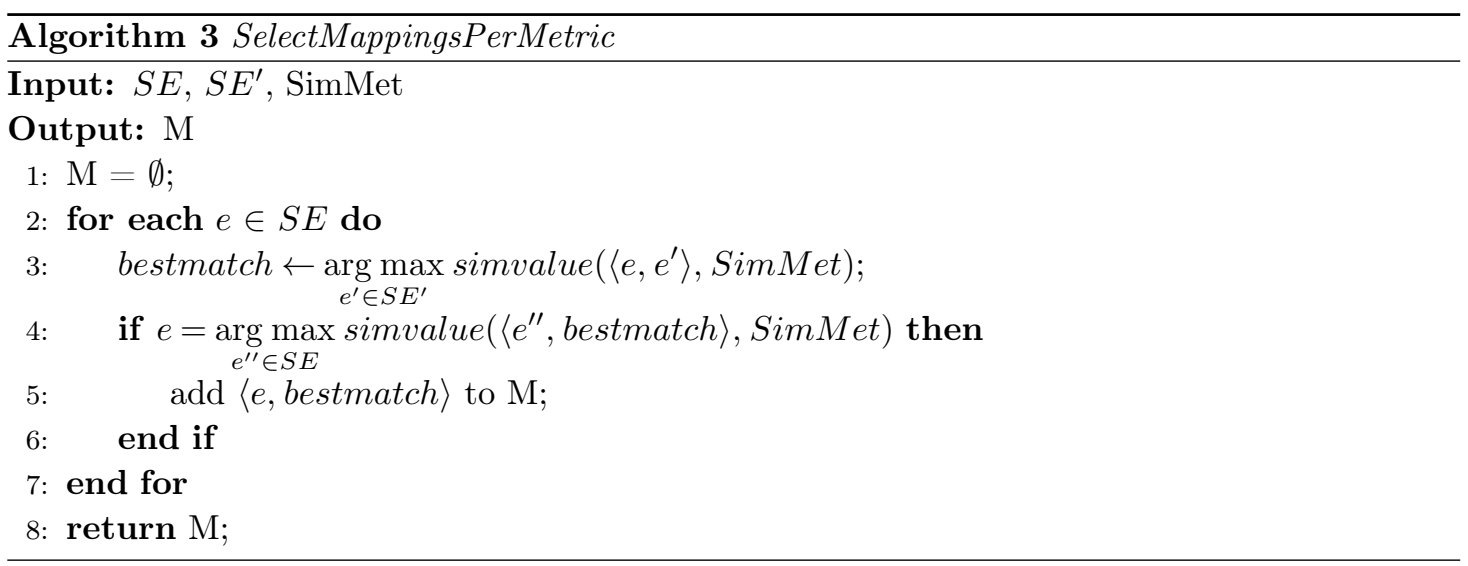

to the accepted ones (from line 1 to line 6 of Algorithm 44. After that, Alin identifies all automatically accepted concept mappings that are in an anti-pattern with other automatically accepted concept mappings (line 7 of Algorithm 44) using the UndoConceptAntiPatterns algorithm (Algorithm 10) and moves them from the accepted mappings to the set of selected mappings. This use of anti-patterns is done to minimize the precision decrease that can occur because of the automatic acceptation of mappings. Subsection 4.3 .3 explains anti-patterns.
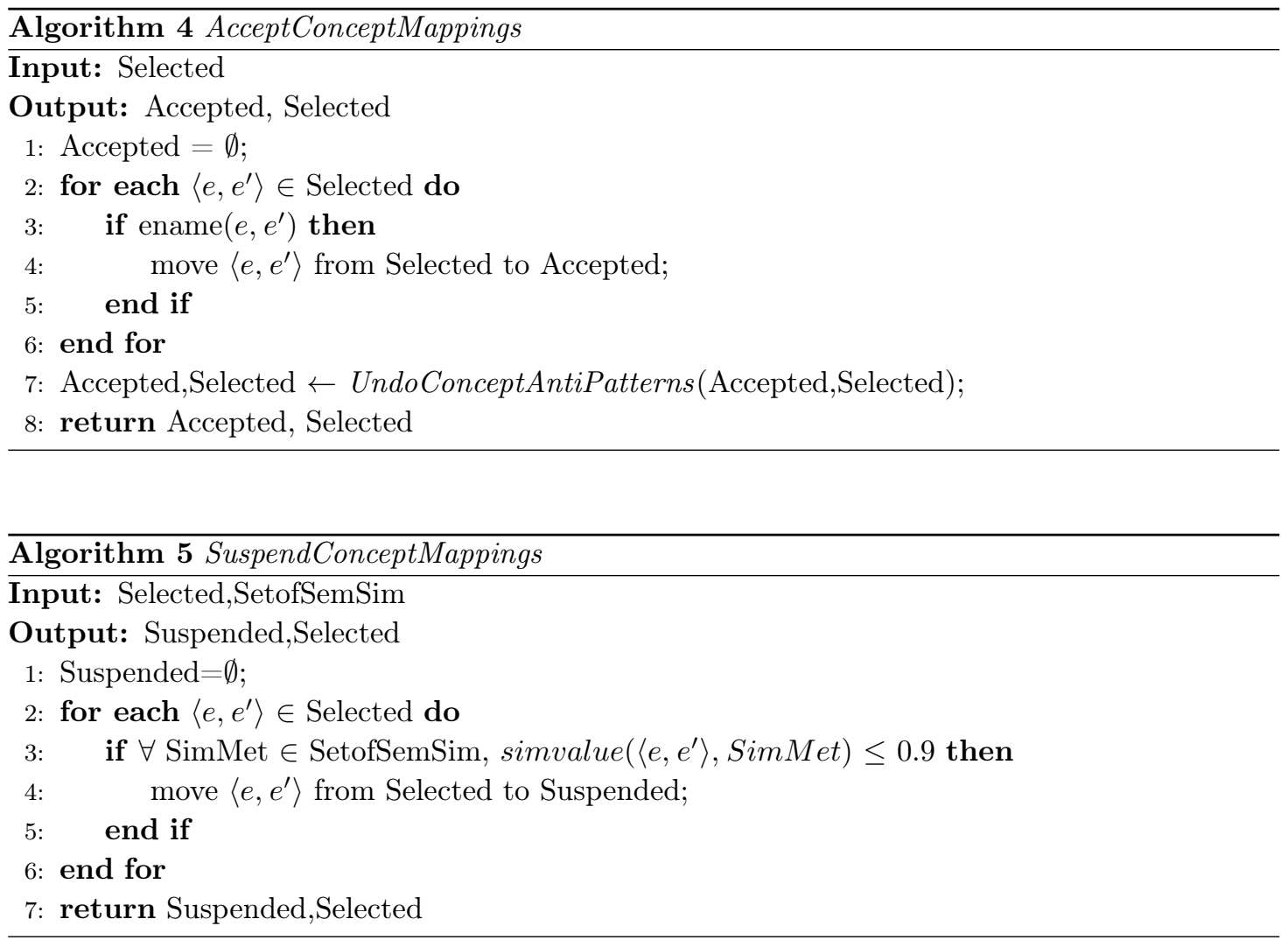

In the selection step, selected mappings less likely to be correct are suspended (line 3 of Algorithm 1), using the SuspendConceptMappings algorithm (Algorithm 5). The SuspendConceptMappings algorithm identifies all selected mappings in which the two concepts have all semantic similarity metric values under a threshold (here 0.9, see Section 5.2) and suspends them. The similarity metrics used in this technique are chosen by the user, e.g., Resnick, Jiang-Conrath or Lin. The suspended mappings can become selected again after interaction with the expert, 
during the propagation step (Subsection 4.3.2), by the use of the UnsuspendConceptMappings algorithm (Algorithm 7).

Subsection 4.3 .2 describes the propagation of the accepted mappings.

\subsubsection{Propagation Step}

The Alin propagation step (from line 4 to line 15 of Algorithm 1) describes the activities to choose the selected mapping to receive expert feedback, the feedback itself, and the feedback propagation.

At the beginning, ALIN propagates the automatically accepted mappings (line 5 of the Algorithm 11 in the same way as it does with the mappings accepted by the expert.

At each iteration in the propagation step (from line 7 to line 15 of the Algorithm 1), Alin chooses among all the selected mappings, the one that has the highest sum of their similarities (line 8 of Algorithm 1). After that, this mapping receives the expert feedback (line 9 of Algorithm 17, where the expert can accept or reject the selected mapping. If the expert accepts the mapping, Alin moves it to the set of accepted mappings (line 12 of Algorithm 1) and propagates its effects (line 13 of Algorithm 11) through the PropagateAcceptedMapping algorithm (Algorithm 6).

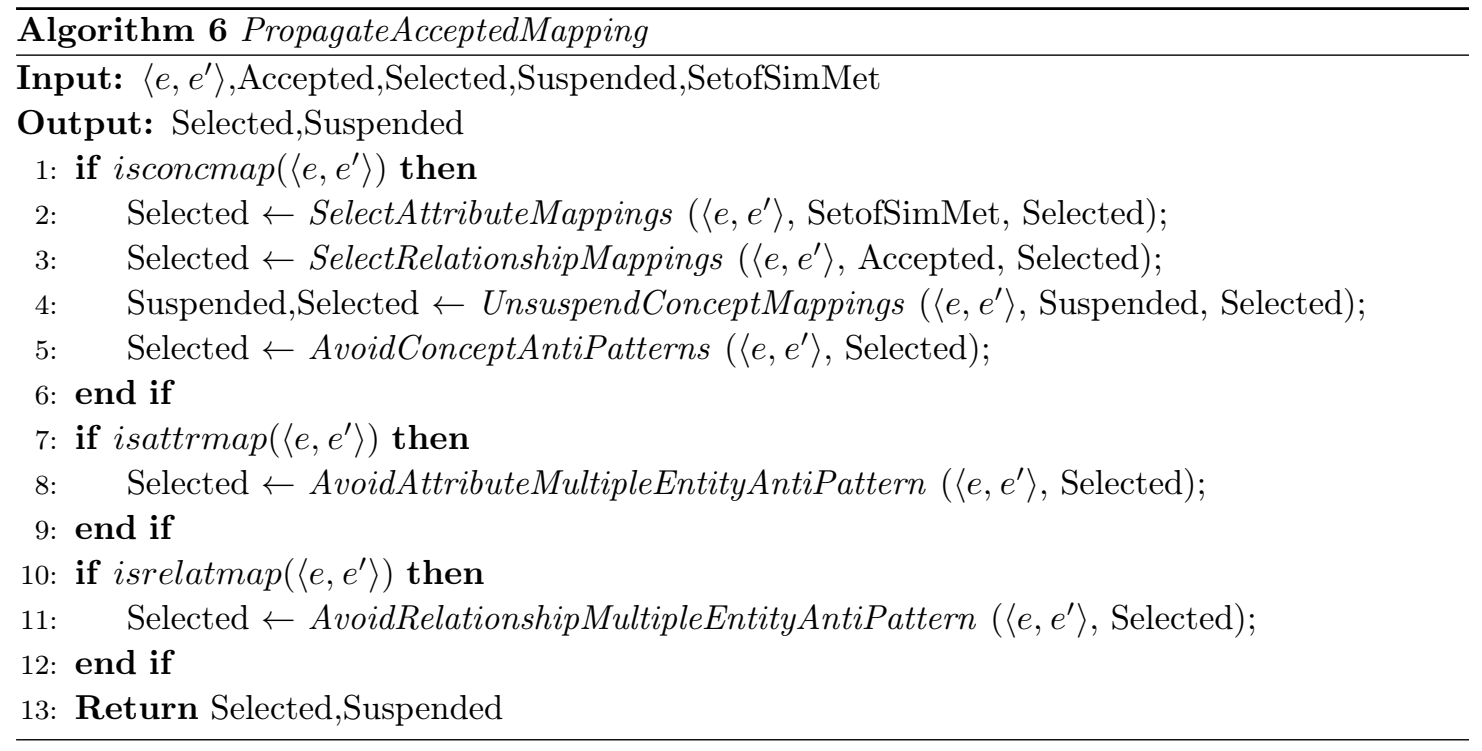

In the propagation step, ALIN only accepts mappings that have received positive feedback from the expert. So, if the expert does not make mistakes, the monotonic growth of the recall and the precision in the propagation step is guaranteed.

The techniques used to propagate the effects of the expert feedback are described in this subsection. The first three techniques described are structural ones that select new mappings. A structural technique takes, as input, one or more mappings, and it generates, as output, mappings structurally related to them.

The UnsuspendConceptMappings algorithm (Algorithm 7) implements the first structural technique. This algorithm assumes that concept mappings are more prone to be true if the superconcepts of both concepts in the mapping are in an accepted mapping. This algorithm, at each iteration, identifies all suspended mappings that are formed by subconcepts of the concepts of the accepted mappings and unsuspend them, i.e., they become again selected mappings. We implemented this technique in a prior version of ALIN: $A L I N_{\text {Syn }}$ (Da Silva et al., 2017).

So far, the ALIN approach has only taken into account concept mappings. With the second structural technique, implemented by the SelectAttributeMappings algorithm (Algorithm 8), attribute mappings of the accepted mappings were selected, i.e., once the expert accepts a concept mapping, ALIN selects some mappings between the attributes of its concepts. 


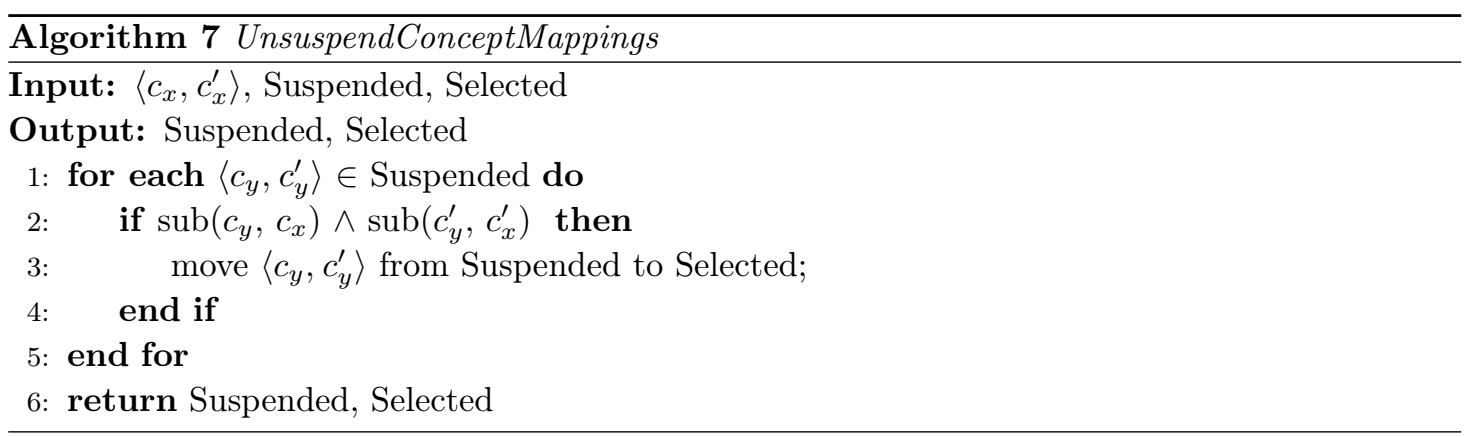

To develop the SelectAttributeMappings algorithm, we have assumed that attribute mappings are more prone to be a mapping between attributes of concepts if their concepts are in an accepted mapping. To reduce the search space to the SelectAttributeMappings algorithm, Alin (line 4 of the Algorithm 8) used the SelectMappingsPerMetric algorithm (Algorithm 3). We implemented this technique in a prior version of Alin: $A L I N_{\text {Attr }}$ (Da Silva, Revoredo, Baião and Euzenat, 2018).

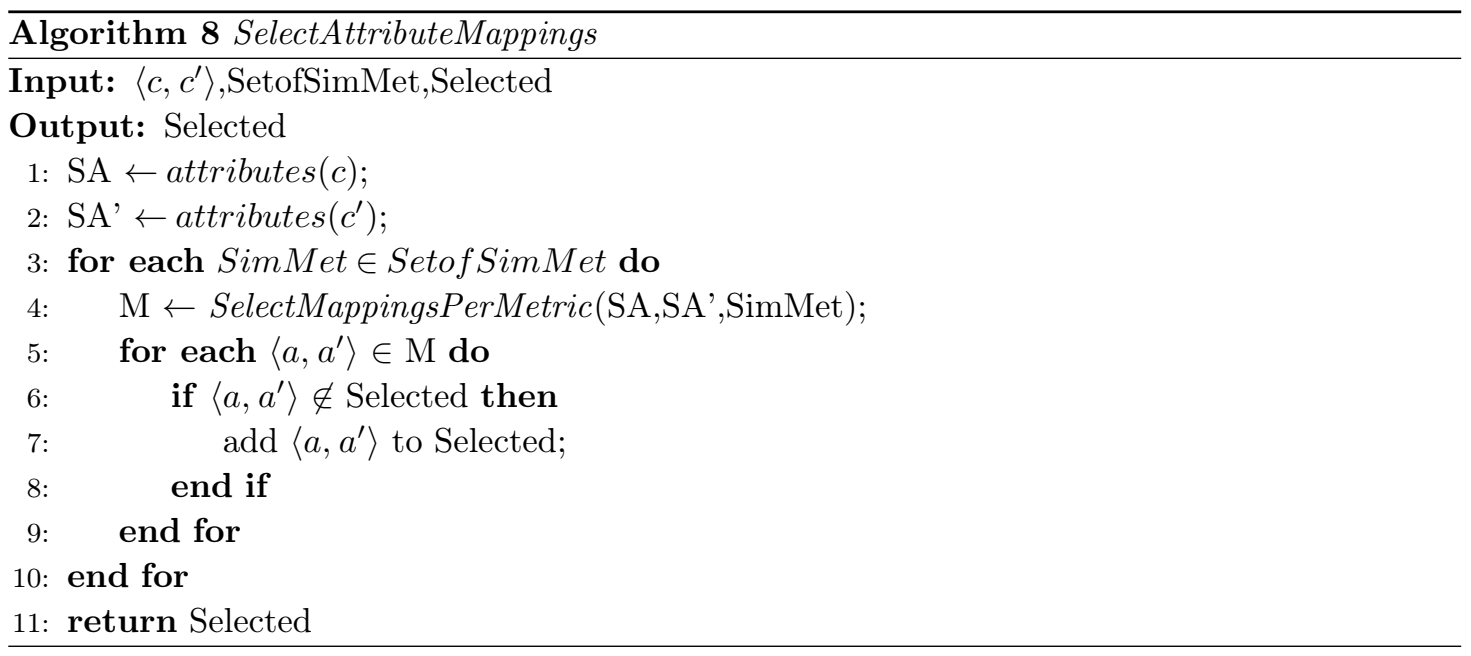

To develop the third structural technique, implemented by the SelectRelationshipMappings algorithm (Algorithm 9), we have assumed that relationships are more prone to be in a mapping if they are relationships between the concepts of two accepted concept mappings. We have also assumed that relationships between subconcepts of these concepts are more prone to be in a mapping too. These situations in which the SelectRelationshipMappings algorithm selects relationship mappings are called triggers for the selection of relationship mappings (Definition 4.1 .

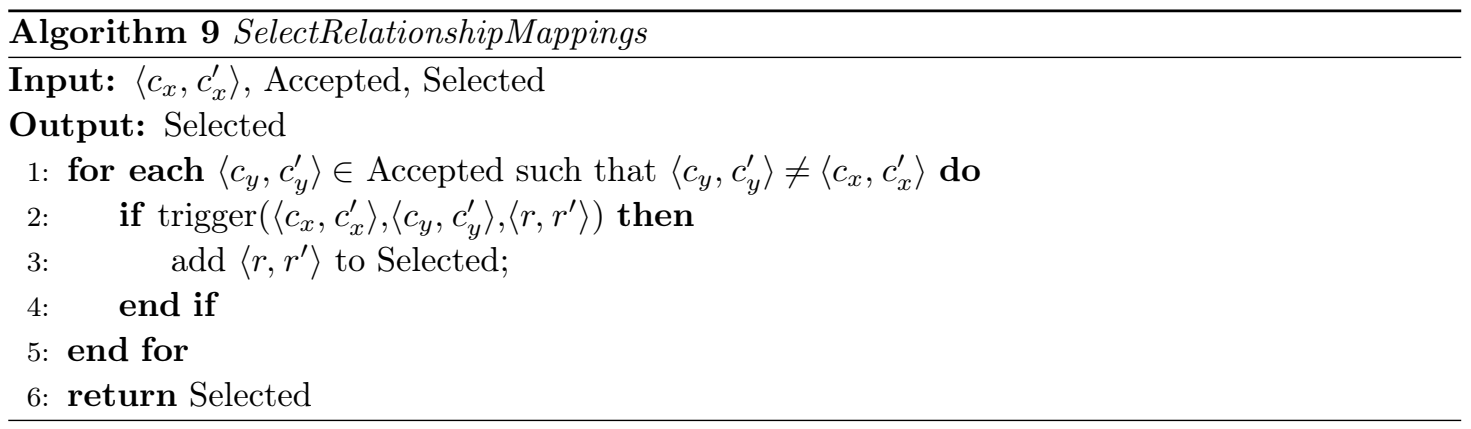


Definition 4.1 (Triggers) Given $\left\langle c_{1}, c_{1}^{\prime}\right\rangle$ and $\left\langle c_{2}, c_{2}^{\prime}\right\rangle$, the following conditions:

- $\quad r$ concept $\left(r, c_{1}, c_{2}\right) \wedge \operatorname{rconcept}\left(r^{\prime}, c_{1}^{\prime}, c_{2}^{\prime}\right)$

- $\operatorname{sub}\left(c_{3}, c_{1}, 2\right) \wedge \operatorname{rconcept}\left(r, c_{3}, c_{1}\right) \wedge \operatorname{rconcept}\left(r^{\prime}, c_{1}^{\prime}, c_{2}^{\prime}\right)$

- $\operatorname{sub}\left(c_{3}^{\prime}, c_{1}^{\prime}, 2\right) \wedge \operatorname{sub}\left(c_{3}, c_{1}, 2\right) \wedge \operatorname{rconcept}\left(r^{\prime}, c_{3}^{\prime}, c_{1}^{\prime}\right) \wedge \operatorname{rconcept}\left(r, c_{3}, c_{1}\right)$

are called triggers between $\left\langle c_{1}, c_{1}^{\prime}\right\rangle$ and $\left\langle c_{2}, c_{2}^{\prime}\right\rangle$ for the selection of the relationship mapping $\left\langle r, r^{\prime}\right\rangle$.

\subsubsection{Mapping Anti-patterns}

An ontology may have construction constraints, such as a concept cannot be equivalent to its superconcept. An alignment may have other constraints like, for example, an entity of ontology $O$ cannot be equivalent to two entities of the ontology $O^{\prime}$. A mapping anti-pattern is a combination of mappings that generates a problematic alignment, i.e., a logical inconsistency or a violated constraint.

ALIN uses three mapping anti-patterns empirically identified by Guedes (Guedes et al. 2014b) (Definitions 4.2 to 4.4 , extracted from the results of ontology matching tools evaluated by OAEI (Guedes et al. 2014a).

The multiple-entity anti-pattern (Definition 4.2 applies when a single entity does not participate in two mappings (Figure 3 (a)). The cross mapping anti-pattern (Definition 4.3 . applies when no subconcept can be equivalent to its superconcept. This mapping anti-pattern applies when a concept $c_{1}$ is a subconcept of the concept $c_{2}$ and $c_{1}^{\prime}$ is subconcept of the concept $c_{2}^{\prime}$ and $c_{1}$ is in a mapping with $c_{2}^{\prime}$ and $c_{2}$ is in a mapping with th $c_{1}^{\prime}$ (see Figure 3 (b)). The disjunction and generalization anti-pattern (Definition 4.4 applies when a pair of concepts of an ontology that are subconcept and superconcept are in mappings with two disjoint concepts of the other ontology (Figure $3(\mathrm{c})$ ).

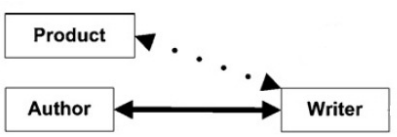

(a)

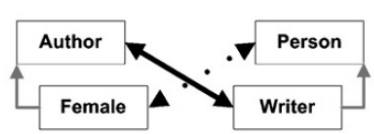

(b)

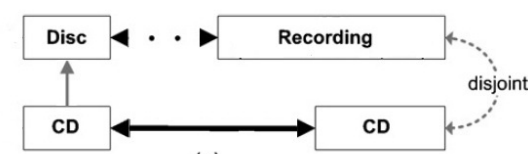

(c)

Figure 3 The three mapping anti-pattern used in Alin. (a) multiple-entity anti-pattern. (b) cross mapping anti-pattern. (c) disjunction and generalization anti-pattern. Based on (Jean-Mary et al., 2009)

Definition 4.2 (Multiple-entity anti-pattern) If the entities $e_{1}, e_{1}^{\prime}$ and $e_{2}^{\prime}$ of mappings $\left\langle e_{1}, e_{1}^{\prime}\right\rangle$ and $\left\langle e_{1}, e_{2}^{\prime}\right\rangle$ occur in the situation described by the formula entity $\left(e_{1}, O\right) \wedge$ entity $\left(e_{1}^{\prime}, O^{\prime}\right) \wedge$ entity $\left(e_{2}^{\prime}, O^{\prime}\right)$, then $\left\langle e_{1}, e_{1}^{\prime}\right\rangle$ and $\left\langle e_{1}, e_{2}^{\prime}\right\rangle$ are said to be in a multiple-entity anti-pattern.

Definition 4.3 (Cross mapping anti-pattern) If the concepts $c_{1}, c_{2}, c_{1}^{\prime}$ and $c_{2}^{\prime}$ of mappings $\left\langle c_{2}, c_{1}^{\prime}\right\rangle$ and $\left\langle c_{1}, c_{2}^{\prime}\right\rangle$ occur in the situation described by the formula concept $\left(c_{1}, O\right) \wedge$ $\operatorname{concept}\left(c_{1}^{\prime}, O^{\prime}\right) \wedge \operatorname{concept}\left(c_{2}, O\right) \wedge \operatorname{concept}\left(c_{2}^{\prime}, O^{\prime}\right) \wedge \operatorname{sub}\left(c_{1}, c_{2}\right) \wedge \operatorname{sub}\left(c_{1}^{\prime}, c_{2}^{\prime}\right)$, then $\left\langle c_{2}, c_{1}^{\prime}\right\rangle$ and $\left\langle c_{1}, c_{2}^{\prime}\right\rangle$ are said to be in a cross mapping anti-pattern.

Definition 4.4 (Disjunction and Generalization anti-pattern) If the concepts $c_{1}, c_{2}$, $c_{1}^{\prime}$ and $c_{2}^{\prime}$ of mappings $\left\langle c_{1}, c_{1}^{\prime}\right\rangle$ and $\left\langle c_{2}, c_{2}^{\prime}\right\rangle$ occur in the situation described by the formula concept $\left(c_{1}, O\right) \wedge \operatorname{concept}\left(c_{1}^{\prime}, O^{\prime}\right) \wedge \operatorname{concept}\left(c_{2}, O\right) \wedge \operatorname{concept}\left(c_{2}^{\prime}, O^{\prime}\right) \wedge \operatorname{sub}\left(c_{1}, c_{2}\right) \wedge$ $\operatorname{dis}\left(c_{1}^{\prime}, c_{2}^{\prime}\right)$, then $\left\langle c_{1}, c_{1}^{\prime}\right\rangle$ and $\left\langle c_{2}, c_{2}^{\prime}\right\rangle$ are said to be in a disjunction and generalization mapping anti-pattern.

ALIN takes advantage of the fact that if a correct mapping is in a mapping anti-pattern with another mapping, the other mapping is wrong. It uses (line 7 of Algorithm 44 the UndoConceptAntiPatterns algorithm (Algorithm 10) to identify automatically accepted concept mappings that are in mapping anti-patterns with others automatically accepted concept mappings 
and moves these mappings from the set of accepted mappings to the set of selected mappings. Alin (line 5 of Algorithm 6) uses the AvoidConceptAntiPatterns algorithm (Algorithm 11) to identify those selected concept mappings that are in a mapping anti-patterns with an accepted concept mapping and removes them from the set of selected mappings. Alin (lines 8 and 11 of Algorithm 6) uses the AvoidAttributeMultipleEntityAntiPattern algorithm (Algorithm 12) and the AvoidRelationshipMultipleEntityAntiPattern algorithm (Algorithm 13) to identify selected attribute and relationship mappings that are in the multiple-entity anti-pattern with an accepted mapping and removes them from the set of selected mappings.
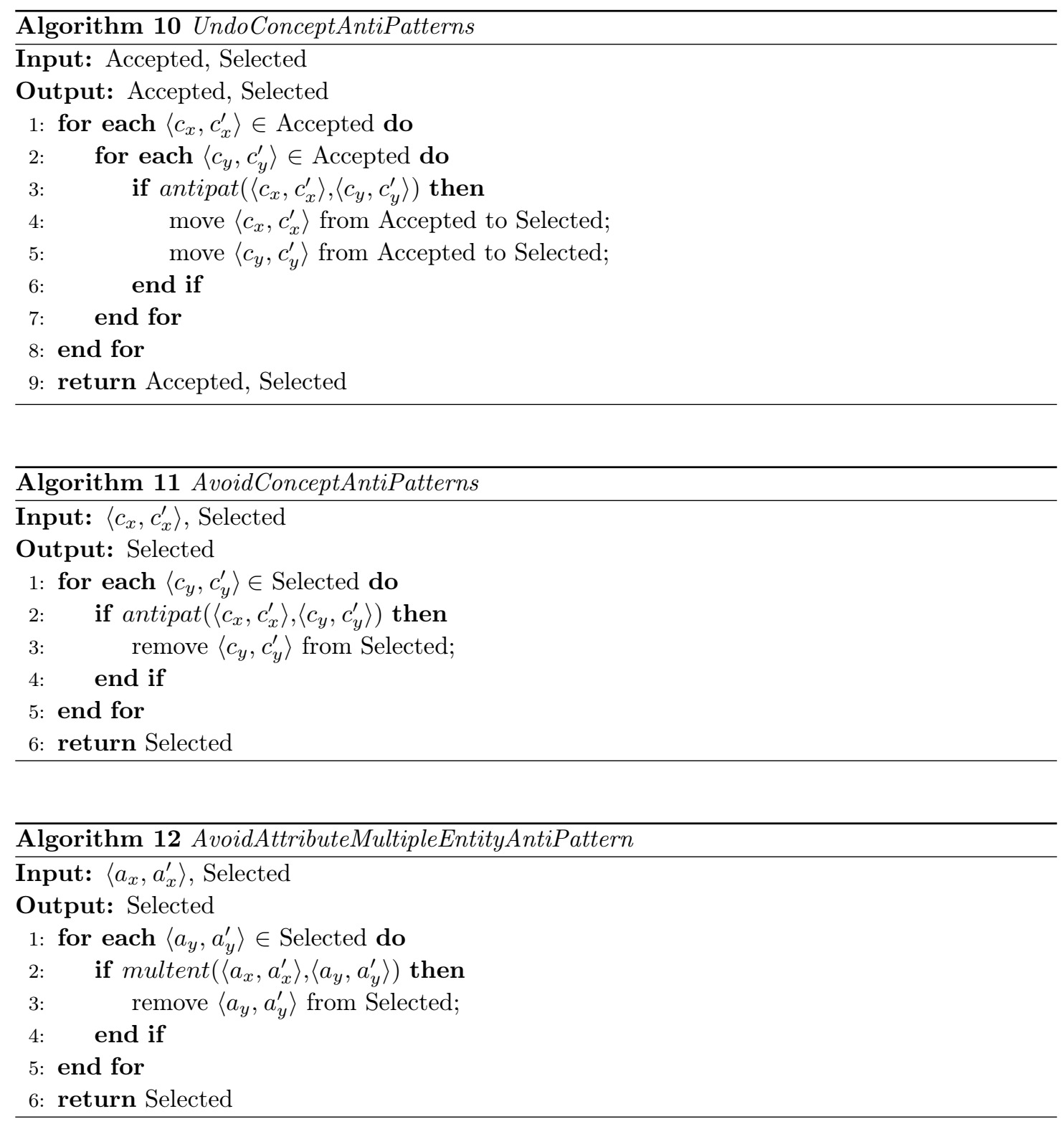

\section{Evaluation overview}

Alin is implemented in Java using the following APIs: Stanford CoreNLP API (Manning et al., 2014 ) to put a word in canonical form; Simmetrics API (Surhone et al., 2010), for string-based similarity metrics; HESML API (Lastra-Díaz et al., 2017), for Wordnet (Fellbaum, 1998) based linguistic metrics; the OWL API for handling ontologies written in OWL, and the Alignment API (David et al. 2011) to deal with alignments. 


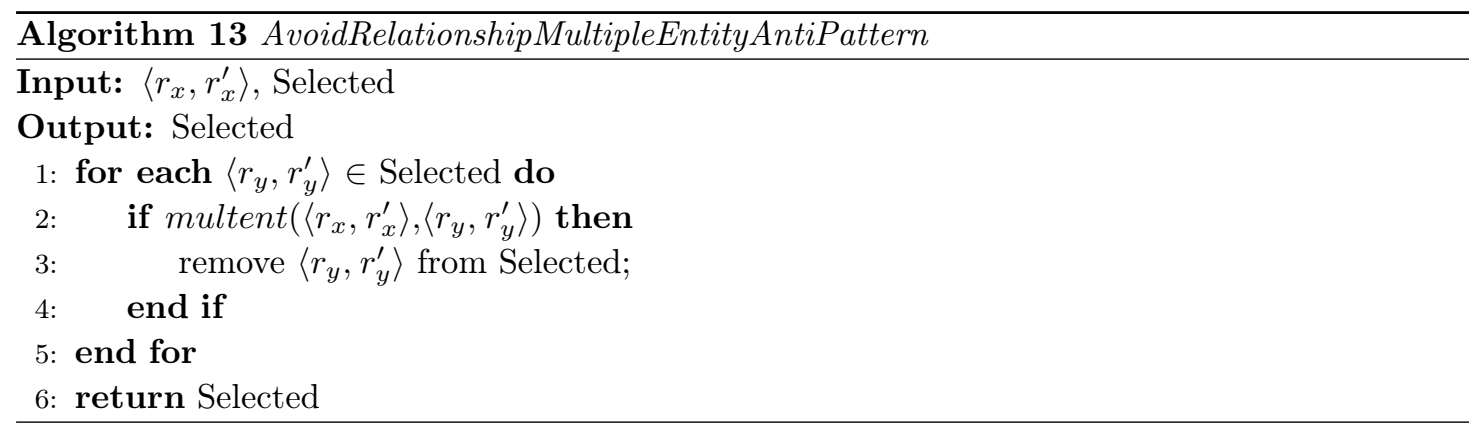

In the executions described in this section, ALIN receives as input parameters six similarity metrics. We based the process of choosing the similarity metrics used by ALIN on the result of these metrics in assessments (Petrakis et al. 2006, Cheatham and Hitzler, 2013). We chose Jaccard, Jaro-Wrinkler, and n-gram lexical metrics and the Resnick, Jiang-Conrath and Lin semantic metrics. Resnick, Jiang-Conrath, and Lin are metrics that require a taxonomy to be computed, this taxonomy is provided by Wordnet (Fellbaum, 1998).

The Ontology Alignment Evaluation Initiative (OAEI) is a coordinated international initiative, which organizes the evaluation of ontology matching systems. Its main goal is to compare systems and algorithms openly and on the same basis, to allow anyone to draw conclusions about the best matching strategies. OAEI provides ontologies for various domains (data sets). The ontologies of data sets can have three types of entities: concepts (classes), data properties (attributes), and object properties (relationships). OAEI provides reference alignments, which are alignments that contains the mappings that are believed to be correct, for each ontology pair in a data set.

To evaluate ALIN, we followed the same protocol as the OAEI 2018 interactive matching track (Algergawy et al., 2018) in which Alin participated (Da Silva, Revoredo and Baião, 2018). An interactive matcher is run and the reference alignment is used to simulate the expert answering. At each interaction, up to three selected mappings can be submitted to the expert, as long as each selected mapping has one entity in common with another selected mapping in the interaction (Faria, 2016). The quality of an alignment generated by a matching approach is generally measured by the F-measure, which is the harmonic mean between recall and precision. When the ontology matching process is interactive another quality metric occurs, it is the number of interactions with the expert. In Tables 4 to 11 hereafter, 'Total Requests' is the number of interactions with the expert.

The OAEI 2018 interactive matching track (Algergawy et al., 2018) is based on two data sets: Conference and Anatomy.

The Conference data set consists of 7 ontologies, resulting in a total of 21 ontology pairs. There are 125,860 possible mappings when we take into account only the mappings of the same entity type among all 21 ontology pairs. Alin takes into account only the mappings of the same entity type. Among the 21 ontology pairs of the Conference data set there are 305 mappings in the reference alignments.

The Anatomy data set that contains two ontologies, one with the mouse anatomy and another with the human anatomy. The number of possible mappings between the two ontologies is $9,066,176$. There are 1,516 mappings in the reference alignment.

\subsection{Evaluation Criteria}

We measure efficiency in the use of expert feedback as the ratio between the F-measure gain over the baseline $\left(A L I N_{N o R e l}\right)$ and the additional number of expert answers.

We raise the following research questions to evaluate if the techniques used in ALIN help to achieve the objective to generate a better alignment: 
RQ1: Does the SelectRelationshipMappings algorithm increases the F-measure of the generated alignment?

RQ2: Is the expert feedback more efficiently used when it assists the SelectRelationshipMappings algorithm?

RQ3: Does the interactive use of mapping anti-patterns decrease the number of interactions with the expert?

RQ4: Does the use of the techniques described in this paper generates a final alignment with quality and number of interactions compatible with the state-of-the-art proposals?

Table 3 Alin versions

\begin{tabular}{|c|c|c|c|}
\hline ALin version & $\begin{array}{l}\text { Use the } \\
\text { SelectRelationshipMappings } \\
\text { algorithm }\end{array}$ & $\begin{array}{c}\text { Use the expert } \\
\text { feedback to assist the } \\
\text { SelectRelationshipMappings } \\
\text { algorithm }\end{array}$ & $\begin{array}{c}\text { Use } \\
\text { anti-patterns }\end{array}$ \\
\hline$A L I N_{N o R e l}$ & No & - & Yes \\
\hline$A L I N_{A u t}$ & Yes & $\mathrm{No}$ & Yes \\
\hline$A L I N_{N o A P}$ & Yes & Yes & $\mathrm{No}$ \\
\hline ALIN & Yes & Yes & Yes \\
\hline
\end{tabular}

In order to evaluate the benefit of the proposed solutions, we developed several versions of Alin:

Alin is the full version of Alin. It uses only the accepted mappings as input of the SelectRelationshipMappings algorithm, i.e., it uses the expert feedback to assist the SelectRelationshipMappings algorithm.

$A L I N_{N o A P}$ is the full ALIN without using anti-patterns. Comparing its results with the results of full ALIN allows answering to RQ3.

$A L I N_{\text {Aut }}$ uses all the automatically selected concept mappings as input to the SelectRelationshipMappings algorithm, i.e., the line 3 of the PropagateAcceptedMapping algorithm (Algorithm 6) is removed and the AutomaticallySelectRelationshipMappings algorithm (Algorithm 14 is called between the lines 1 and 2 of Algorithm 1. Since it uses the automatically selected concept mappings as input, it does not use the expert feedback to the SelectRelationshipMappings algorithm. Comparing the results of its execution with the results of $A L I N_{N o R e l}$ allows answering to RQ1. Comparing the results of its execution with the the results of $A L I N_{N o R e l}$ and the results of full Alin allows answering to RQ2.

$A L I N_{N o R e l}$ does not use the SelectRelationshipMappings algorithm, i.e., line 3 and lines from 10 to 12 are removed from the PropagateAcceptedMapping algorithm (Algorithm66. Comparing the results of its execution with the results of $A L I N_{A u t}$ allows answering to RQ1. Comparing the results of its execution with the the results of $A L I N_{A u t}$ and the results of full Alin allows answering to RQ2.

Table 3 shows the characteristics of ALIN versions.

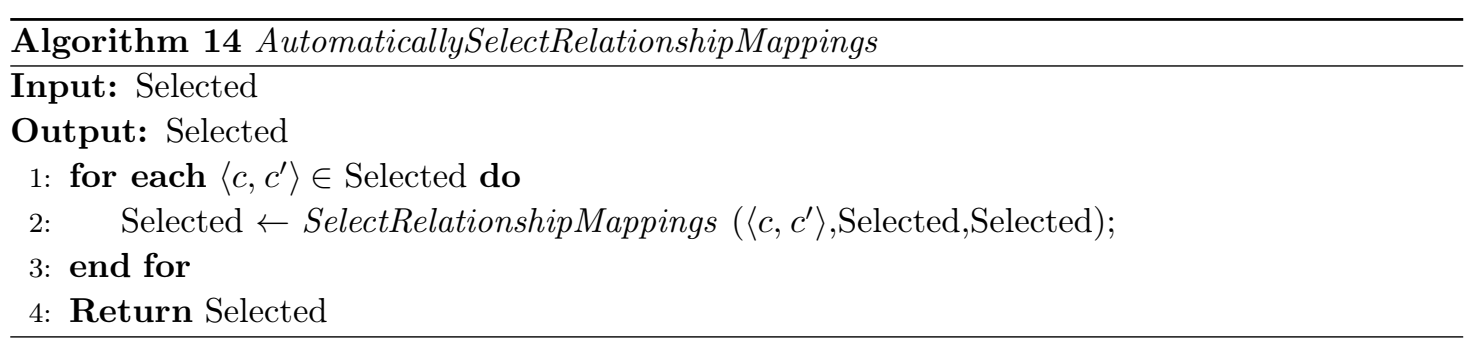




\subsection{Parameter Tunning}

In the SelectRelationshipMappings algorithm, we searched for the relationship mappings of the subconcepts of the concepts of two accepted concept mappings. During the creation of this algorithm, we found out that if we fixed a maximum depth of the subconcepts that we searched, the number of relationship mappings that the algorithm would select decreased. So the number of interactions with the expert. The depth of 2 was where we found the maximum recall, and we set this value for ALIN. From this value, we found only relationships mappings that were not accepted by the expert (Table 4 .

Table 4 Comparison between Alin executions with different maximum depths of the subconcepts using Conference data set

\begin{tabular}{ccccc}
\hline $\begin{array}{c}\text { Maximum Depth } \\
\text { of the Subconcepts }\end{array}$ & Total Requests & Precision & F-measure & Recall \\
\hline \hline $\begin{array}{c}\text { With no } \\
\text { Relationships Mappings }\end{array}$ & 246 & 0.918 & 0.793 & 0.698 \\
0 & 251 & 0.919 & 0.798 & 0.705 \\
1 & 254 & 0.919 & 0.802 & 0.711 \\
2 & 276 & 0.921 & 0.809 & 0.721 \\
3 & 295 & 0.921 & 0.809 & 0.721 \\
4 & 310 & 0.921 & 0.809 & 0.721 \\
5 & 321 & 0.921 & 0.809 & 0.721 \\
\hline
\end{tabular}

We modified the SuspendConceptMapping algorithm in ALIN. We removed, in $\operatorname{ALIN}_{\text {Syn }}$ (Da) Silva et al. 2017), all mappings from the set of selected mappings in which the two mapping concepts were not semantically equivalent, i.e., if all of the semantic similarity metrics were less than 1. In Alin, using the Conference data set, we did several executions changing the minimum value of similarity, and we found that the value of 0.9 reached the best result, so in ALIN, we fixed the value in 0.9 (Table 5). Although we have found the value by running ALIN with the Conference data set, we used the same value with the Anatomy data set.

Since the parameter values were empirically set, further study would be required to make them more general.

Table 5 Comparison between Alin executions with different minimum semantic similarity values of the entities using the Conference data set

\begin{tabular}{ccccc}
\hline $\begin{array}{c}\text { Minimum Semantic Similarity } \\
\text { between the Entities }\end{array}$ & Total Requests & Precision & F-measure & Recall \\
\hline \hline 1.0 & 257 & 0.917 & 0.789 & 0.692 \\
0.9 & 276 & 0.921 & 0.809 & 0.721 \\
0.8 & 300 & 0.921 & 0.809 & 0.721 \\
0.7 & 310 & 0.920 & 0.805 & 0.715 \\
\hline
\end{tabular}

\subsection{Analysis of the results}

We made executions with the three versions of Alin for both the Conference data set and the Anatomy data set. The execution of $A L I N_{A u t}$, for the Conference data set, using the SelectRelationshipMappings algorithm generates an alignment with higher F-measure relatively to $A L I N_{N o R e l}$. Thus, this execution responds positively to RQ1.

With all the selected mappings as input to the SelectRelationshipMappings algorithm in $A L I N_{A u t}$, there is an increase of $1.02 \%$ in F-measure, but with $29.26 \%$ more interactions 
Table 6 Comparison between different ontology matching executions with Conference data set

\begin{tabular}{|c|c|c|c|c|c|c|c|}
\hline & $\begin{array}{c}\text { Total } \\
\text { Req }\end{array}$ & Prec & $\mathrm{F}$ & Rec & $\begin{array}{c}\text { Increase } \\
\text { in } \\
\text { Total } \\
\text { Requests } \\
\text { relative } \\
\text { to } \\
A L I N_{N o R e l}\end{array}$ & $\begin{array}{l}\text { Increase } \\
\text { in } \\
\text { F-measure } \\
\text { relative } \\
\text { to } \\
A L I N_{N o R e l}\end{array}$ & $\begin{array}{c}\text { Efficiency } \\
\text { relative } \\
\text { to } \\
A L I N_{N o R e l}\end{array}$ \\
\hline$A L I N_{N o R e l}$ & 246 & 0.918 & 0.793 & 0.698 & - & - & - \\
\hline$A L I N_{A u t}$ & 318 & 0.921 & 0.811 & 0.725 & 29.26 & 1.02 & 0.011 \\
\hline ALIN & 276 & 0.921 & 0.809 & 0.721 & 12.19 & 1.02 & 0.027 \\
\hline
\end{tabular}

Table 7 Comparison between different ontology matching executions with Anatomy data set

\begin{tabular}{|c|c|c|c|c|c|c|c|}
\hline & $\begin{array}{c}\text { Total } \\
\text { Req }\end{array}$ & Prec & $\mathrm{F}$ & Rec & $\begin{array}{c}\text { Increase } \\
\text { in } \\
\text { Total } \\
\text { Requests } \\
\text { relative } \\
\text { to } \\
A L I N_{N o R e l} \\
\end{array}$ & $\begin{array}{l}\text { Increase } \\
\text { in } \\
\text { F-measure } \\
\text { relative } \\
\text { to } \\
A L I N_{N o R e l}\end{array}$ & $\begin{array}{c}\text { Efficiency } \\
\text { relative } \\
\text { to } \\
A L I N_{N o R e l}\end{array}$ \\
\hline$A L I N_{N o R e l}$ & 602 & 0.994 & 0.902 & 0.826 & - & - & - \\
\hline$A L I N_{A u t}$ & 602 & 0.994 & 0.902 & 0.826 & - & - & - \\
\hline Alin & 602 & 0.994 & 0.902 & 0.826 & - & - & - \\
\hline
\end{tabular}

with the expert relative to $A L I N_{N o R e l}$. With the use of accepted mappings as input to the SelectRelationshipMappings algorithm in Full Alin, we got almost the same increase in F-measure, but with only $12.19 \%$ more interactions relative to $A L I N_{N o R e l}$. So, concerning $A L I N_{N o R e l}$, the Full ALIN allows more efficiency in the use of the expert feedback than $A L I N_{A u t}$. Thus, this execution responds positively to RQ2.

With the use of the Anatomy data set, all versions achieved the same alignment, and thus the same F-measure (Table 7). This is because there are no relationships in the ontologies of the Anatomy data set, hence the SelectRelationshipMappings algorithm brings no benefit.

Thus, the executions show that we can only respond positively to both RQ1 and RQ2 if that involved ontologies have relationships.

The interactive use of mapping anti-patterns by the ALIN approach decreases the number of interactions with the expert without decreasing the F-measure (Tables 8 and 9), which respond positively to the RQ3.

Table 8 Comparison between ALIN executions with and without mapping anti-patterns using the Conference data set

\begin{tabular}{ccccc}
\hline & Total Requests & Precision & F-measure & Recall \\
\hline \hline$A L I N_{\text {NoAP }}$ & 329 & 0.921 & 0.809 & 0.721 \\
ALIN & 276 & 0.921 & 0.809 & 0.721 \\
\hline
\end{tabular}


Table 9 Comparison between Alin executions with and without mapping anti-patterns using the Anatomy data set

\begin{tabular}{ccccc}
\hline & Total Requests & Precision & F-measure & Recall \\
\hline \hline$A L I N_{\text {NoAP }}$ & 679 & 0.993 & 0.903 & 0.828 \\
ALIN & 602 & 0.994 & 0.902 & 0.826 \\
\hline
\end{tabular}

Table 10 Comparison between OAEI 2018 interactive matching track tools using Conference data set with $100 \%$ hit rate (Algergawy et al. 2018)

\begin{tabular}{ccccc}
\hline & Total Requests & Precision & F-measure & Recall \\
\hline \hline ALIN & 276 & $\mathbf{0 . 9 2 1}$ & $\mathbf{0 . 8 0 9}$ & $\mathbf{0 . 7 2 1}$ \\
AML & 270 & 0.912 & 0.799 & 0.711 \\
LogMap & 82 & 0.886 & 0.723 & 0.61 \\
XMap & $\mathbf{1 6}$ & 0.719 & 0.666 & 0.62 \\
\hline
\end{tabular}

Table 11 Comparison between OAEI 2018 interactive matching track tools using Anatomy data set with $100 \%$ hit rate (Algergawy et al. 2018)

\begin{tabular}{ccccc}
\hline & Total Requests & Precision & F-measure & Recall \\
\hline \hline ALIN & 602 & $\mathbf{0 . 9 9 4}$ & 0.902 & 0.826 \\
AML & 240 & 0.964 & $\mathbf{0 . 9 5 6}$ & $\mathbf{0 . 9 4 8}$ \\
LogMap & 388 & 0.982 & 0.909 & 0.846 \\
XMap & $\mathbf{3 5}$ & 0.929 & 0.897 & 0.867 \\
\hline
\end{tabular}

\subsection{Comparison between tools that participated in the OAEI 2018 interactive matching track}

ALIN participated in the OAEI 2018 interactive matching track. OAEI provides a comparison between tool performance in the interactive matching track each year, and it uses the Conference and the Anatomy data sets (Algergawy et al. 2018), as we can see in the tables 10 and 11.

The tools AML, LogMap, and XMAP (Tables 10 and 11) are interactive ontology matching tools which select attribute and relationship mappings, but in a non-interactive way, not taking into account the expert feedback.

In relation of the F-measure, the results show that ALIN generated a high-level result when running the Conference data set (Table 10) and ALIN got a result close to other tools when running the Anatomy data set (Table 11) when the expert hit $100 \%$ of the answers, which answers positively to RQ4.

In relation to number of interactions with the expert, ALIN, when running with the Conference data set, has a number of interactions more compatible with the other tools than with the Anatomy data set. That is because the ontologies of the Conference dataset, contrary to those of Anatomy, contain attributes and relationships, which allows the use of the SelectRelationshipMappings and the SelectAttributeMappings algorithms to improve the set of selected mappings.

When the expert make mistakes, ALIN suffers a sharp fall, relative to other tools (Figures 4 and 5), of the F-measure. This fall is because ALIN uses expert feedback as input to the used techniques, which causes these techniques to generate erroneous results when the expert fails. Besides, these outputs of the techniques serve to modify the set of selected mappings, which causes the set of selected mappings to get worse, that is, correct mappings can come out, and wrong mappings can enter. 


\section{Conference Data Set}

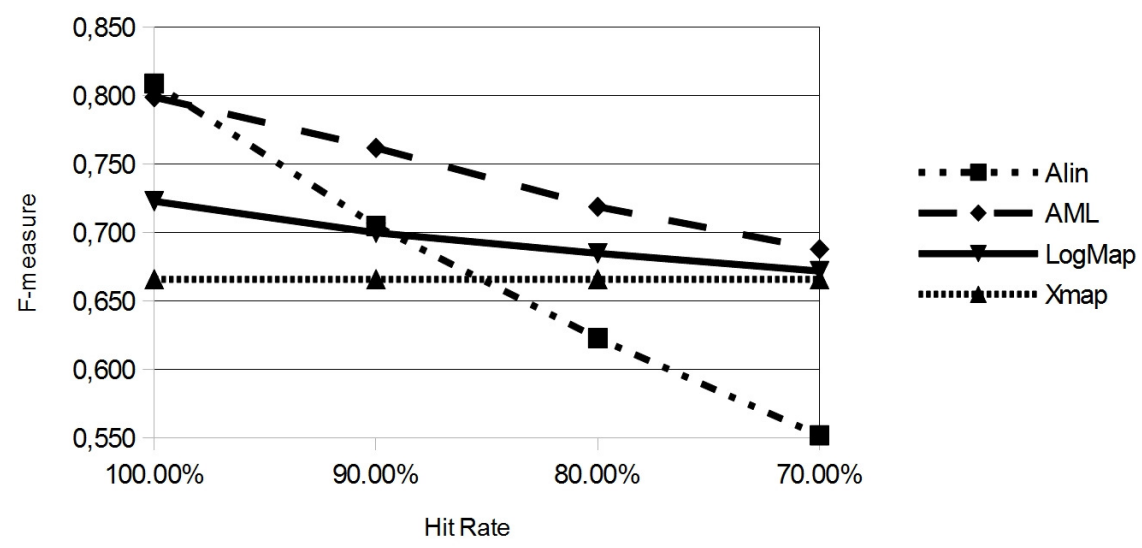

Figure 4 Comparison of execution of several interactive tools with different hit rates - Conference data set

Anatomy Data Set

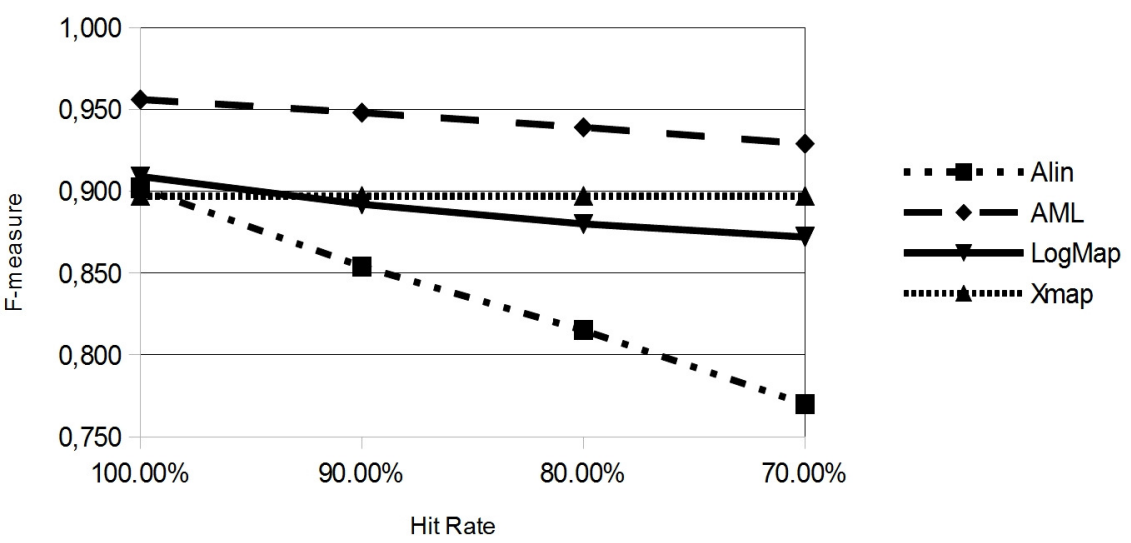

Figure 5 Comparison of execution of several interactive tools with different hit rates - Anatomy data set

\section{Conclusions and Future Works}

Ontology matching plays an important role in the integration of heterogeneous data sources that are described by ontologies. Its purpose is to discover mappings between the entities of at least two ontologies.

Alin contributes to interactive ontology matching techniques through the use of expert feedback for the improvement of the set of selected mappings. It does this by using expert feedback to select relationship mappings and to reject selected mappings through the use of anti-patterns.

We evaluated the various new features of the ALIN approach by comparing different versions of the system. The evaluation performed showed that the use of the expert feedback to improve the set of selected mappings increased the F-measure gain per interaction.

ALIN participated in the interactive ontology matching track of OAEI 2018. The results showed that ALIN generates an alignment with good quality in comparison to other tools, in precision, 
recall and F-measure when the expert never makes mistakes. Hence, in spite of good quality results, the proposed technique is no silver bullet for ontology matching.

The Alin approach had good results when the expert does not make mistake, but as the approach uses expert feedback to select and reject mappings, expert mistakes generate noise for the structural techniques and for the use of mapping anti-patterns. As future work, one interesting direction is to explore how to reduce the negative effects of expert mistakes. In this case, the question of using several experts simultaneously can occur and require specific treatments.

A more informative evaluation to assess the benefits of using the expert feedback to assist the selection of mappings would consist of applying it to the mappings generated by other systems participating in OAEI.

\section{Acknowledgments}

Jérôme Euzenat was partially funded by a CNPq Special Visiting Researcher grant (314782/2014$1)$.

\section{References}

Algergawy, A., Cheatham, M., Faria, D., Ferrara, A., Fundulaki, I., Harrow, I., Hertling, S., Jiménez-Ruiz, E., Karam, N., Khiat, A., Lambrix, P., Li, H., Montanelli, S., Paulheim, H., Pesquita, C., Saveta, T., Schmidt, D., Shvaiko, P., Splendiani, A., Thiéblin, E., Trojahn, C., Vataščinová, J., Zamazal, O. and Zhou, L. (2018), 'Results of the ontology alignment evaluation initiative 2018', CEUR Workshop Proceedings 2288, 76-116.

http://www.dit.unitn.it/〜pavel/om2018/papers/oaei18_paper0.pdf

Balasubramani, B., Taheri, A. and Cruz, I. (2015), User involvement in ontology matching using an online active learning approach, in 'CEUR Workshop Proceedings', Vol. 1545, CEUR-WS, pp. $45-49$.

Belkin, N. J., Bierig, R., Buscher, G., Jose, J. and Teevan, J. (2010), 'Understanding the user - logging and interpreting user interactions in information search and retrieval', Information Retrieval (October 2015), 2009-2010.

Cheatham, M. and Hitzler, P. (2013), String similarity metrics for ontology alignment, in 'Proceedings of the 12th International Semantic Web Conference - Part II', ISWC '13, SpringerVerlag New York, Inc., New York, NY, USA, pp. 294-309.

Chunhua, L., Zhiming, C., Pengpeng, Z., Jian, W., Jie, X. and Tianxu, H. (2015), Improving ontology matching with propagation strategy and user feedback, in 'Seventh International Conference on Digital Image Processing (ICDIP 2015)', Vol. 9631, p. 6.

https://doi.org/10.1117/12.2197167

Cruz, I. F., Loprete, F., Palmonari, M., Stroe, C. and Taheri, A. (2014), Pay-as-you-go multiuser feedback model for ontology matching, in K. Janowicz, S. Schlobach, P. Lambrix and E. Hyvönen, eds, 'Knowledge Engineering and Knowledge Management', Springer International Publishing, Cham, pp. 80-96.

Cruz, I. F., Stroe, C. and Palmonari, M. (2012), Interactive user feedback in ontology matching using signature vectors, in '28th IEEE International Conference on Data Engineering', pp. 1321-1324.

Da Silva, J., Revoredo, K. and Baião, F. A. (2018), ALIN results for OAEI 2018. http://www.dit.unitn.it/〜pavel/om2018/papers/oaei18_paper1.pdf 
Da Silva, J., Revoredo, K., Baião, F. A. and Euzenat, J. (2017), Semantic interactive ontology matching: Synergistic combination of techniques to improve the set of candidate correspondences, in 'OM 2017 - 12th ISWC workshop on ontology matching', Vol. 2032, pp. 13-24. http://disi.unitn.it/〜pavel/om2017/papers/om2017_Tpaper2.pdf

Da Silva, J., Revoredo, K., Baião, F. A. and Euzenat, J. (2018), Interactive Ontology Matching: Using Expert Feedback to Select Attribute Mappings. http://disi.unitn.it/〜pavel/om2018/papers/om2018_LTpaper3.pdf

David, J., Euzenat, J., Scharffe, F. and Trojahn dos Santos, C. (2011), 'The Alignment API 4.0', Semant. web 2(1), 3-10. http://dl.acm.org/citation.cfm?id=2019470.2019474

Duan, S., Fokoue, A. and Srinivas, K. (2010), One size does not fit all: Customizing ontology alignment using user feedback, in P. F. Patel-Schneider, Y. Pan, P. Hitzler, P. Mika, L. Zhang, J. Z. Pan, I. Horrocks and B. Glimm, eds, 'The Semantic Web - ISWC 2010', Springer Berlin Heidelberg, Berlin, Heidelberg, pp. 177-192.

Euzenat, J. and Shvaiko, P. (2013), Ontology Matching - Second Edition, Springer-Verlag.

Faria, D. (2016), 'Using the SEALS Client's Oracle in Interactive Matching'. https://github.com/DanFaria/OAEI\{_\}SealsClient/blob/master/OracleTutorial.pdf

Faria, D., Pesquita, C., Santos, E., Palmonari, M., Cruz, I. F. and Couto, F. M. (2013), The AgreementMakerLight ontology matching system, in R. Meersman, H. Panetto, T. Dillon, J. Eder, Z. Bellahsene, N. Ritter, P. De Leenheer and D. Dou, eds, 'On the Move to Meaningful Internet Systems: OTM 2013 Conferences', Springer Berlin Heidelberg, Berlin, Heidelberg, pp. 527-541.

Fellbaum, C., ed. (1998), WordNet: An electronic lexical database, MIT Press.

Gale, D. and Shapley, L. S. (1962), 'College admissions and the stability of marriage', The American Mathematical Monthly 69(1), 9-15. https://doi.org/10.1080/00029890.1962.11989827

Guedes, A., Baião, F. and Revoredo, K. (2014a), Digging Ontology Correspondence Antipatterns, in 'Proceedings of the 5th Workshop on Ontology and Semantic Web Patterns (WOP2014) co-located with the 13th International Semantic Web Conference (ISWC 2014)', Vol. 1032, pp. $38-48$.

Guedes, A., Baião, F. and Revoredo, K. (2014b), On the Identification and Representation of Ontology Correspondence Antipatterns, in 'Proceedings of the 8th International Workshop on Modular Ontologies co-located with the 8th International Conference on Formal Ontology in Information Systems (FOIS 2014), CEUR Workshop Proceedings', Vol. 1248.

Hertling, S. (2012), Hertuda results for OAEI 2012, in 'Proceedings of the 7th International Conference on Ontology Matching - Volume 946', OM'12, CEUR-WS.org, Aachen, Germany, Germany, pp. 141-144. http://dl.acm.org/citation.cfm?id=2887596.2887607

Irving, R. W., Manlove, D. F. and O'Malley, G. (2009), 'Stable marriage with ties and bounded length preference lists', Journal of Discrete Algorithms 7(2), 213 - 219. Selected papers from the 2nd Algorithms and Complexity in Durham Workshop ACiD 2006. http://www.sciencedirect.com/science/article/pii/S1570866708000683

Jean-Mary, Y. R., Shironoshita, E. P. and Kabuka, M. R. (2009), Ontology matching with semantic verification. 
Jiménez-Ruiz, E. and Grau, B. C. (2011), LogMap: Logic-based and scalable ontology matching, in L. Aroyo, C. Welty, H. Alani, J. Taylor, A. Bernstein, L. Kagal, N. Noy and E. Blomqvist, eds, 'The Semantic Web - ISWC 2011', Springer Berlin Heidelberg, Berlin, Heidelberg, pp. 273-288.

Jiménez-Ruiz, E., Grau, B. C., Zhou, Y. and Horrocks, I. (2012), Large-scale interactive ontology matching: Algorithms and implementation, in 'ECAI 2012 - 20th European Conference on Artificial Intelligence', Vol. 242, pp. 444-449.

Lambrix, P. and Kaliyaperumal, R. (2016), 'A Session-based Ontology Alignment Approach enabling User Involvement', Semantic Web 1, 1-28.

Lastra-Díaz, J. J., García-Serrano, A., Batet, M., Fernández, M. and Chirigati, F. (2017), 'HESML: A scalable ontology-based semantic similarity measures library with a set of reproducible experiments and a replication dataset', Inf. Syst. 66(C), 97-118.

https://doi.org/10.1016/j.is.2017.02.002

Lopes, V., Baião, F. and Revoredo, K. (2015), Alinhamento Interativo de Ontologias Uma Abordagem Baseada em Query-by-Committee, Master's thesis, UNIRIO.

Manning, C. D., Surdeanu, M., Bauer, J., Finkel, J., Bethard, S. J. and McClosky, D. (2014), The Stanford CoreNLP natural language processing toolkit, in 'Association for Computational Linguistics (ACL) System Demonstrations', pp. 55-60.

http://www.aclweb.org/anthology/P/P14/P14-5010

Ngo, D., Bellahsene, Z. and Todorov, K. (2013), Opening the black box of ontology matching, in P. Cimiano, O. Corcho, V. Presutti, L. Hollink and S. Rudolph, eds, 'The Semantic Web: Semantics and Big Data', Springer Berlin Heidelberg, Berlin, Heidelberg, pp. 16-30.

Paulheim, H. and Hertling, S. (2013), WeSeE-match results for OAEI 2013, in 'Proceedings of the 8th International Conference on Ontology Matching - Volume 1111', OM'13, CEUR-WS.org, Aachen, Germany, Germany, pp. 197-202. http://dl.acm.org/citation. cfm?id=2874493.2874513

Paulheim, H., Hertling, S. and Ritze, D. (2013), 'Towards Evaluating Interactive Ontology Matching Tools', Lecture Notes in Computer Science 7882, 31-45.

Petrakis, E. G. M., Varelas, G., Hliaoutakis, A. and Raftopoulou, P. (2006), 'Design and Evaluation of Semantic Similarity Measures for Concepts Stemming from the Same or Different Ontologies object instrumentality', Proceedings of the 4 th Workshop on Multimedia Semantics (WMS) 4, 233-237.

Shi, F., Li, J., Tang, J., Xie, G. and Li, H. (2009), Actively learning ontology matching via user interaction, in A. Bernstein, D. R. Karger, T. Heath, L. Feigenbaum, D. Maynard, E. Motta and K. Thirunarayan, eds, 'The Semantic Web - ISWC 2009', Springer Berlin Heidelberg, Berlin, Heidelberg, pp. 585-600.

Surhone, L. M., Timpledon, M. T. and Marseken, S. F. (2010), SimMetrics, VDM Publishing.

To H., Ichise, R. and Le, H. (2009), An Adaptive Machine Learning Framework with User Interaction for Ontology Matching, in 'Proc. Twenty-first International Joint Conference on Artificial Intelligence'. 